\title{
Electroless Deposition of Confined Copper Layers Based on Selective Activation by Pulsed Laser Irradiation
}

\author{
Rui Zhou*, Tingting Huang, Lijuan Chen, Sijie Chen, Shengdong Lin and Yong Zhuo \\ School of Aerospace Engineering, Xiamen University, 422 Siming South Road, Siming District, \\ Xiamen, Fujian Province, China 361005 \\ *E-mail: rzhou2@xmu.edu.cn
}

\begin{abstract}
Copper circuit was successfully fabricated on non-conductive modified polycarbonate (PC) lightweight plastic substrate by pulsed laser ablation. The modified PC plastic embedded with copper nanoparticles provides a possible route for electroless copper deposition. Copper layer can be selectively confined in the area of pulsed laser activation on the PC substrate. Laser ablation can remove the polymeric substrate material with controlled processing parameters and make embedded copper seeds exposed as self-catalytic centers. In this work, the effects of laser power and scanning speed on the electrical performance of deposited copper layer are investigated. Energy Dispersive $\mathrm{X}$-ray (EDX) is employed to analyze the surface chemistry of activated areas. The dynamic process and deposition mechanism of copper layer on PC surface are studied. The resistivity of the copper layer is strongly dependent on laser power and scanning speed. An optimized resistivity of 15 $\mu \Omega \cdot \mathrm{cm}$ is achieved.
\end{abstract}

DOI: 10.2961/jlmn.2017.02.0021

Keywords: pulsed laser ablation, selective activation, modified polycarbonate, copper deposition, resistivity

\section{Introduction}

Lightweight technology is now a raising focus which has great potentials in a wide range of applications in portable microelectronics, wearable device, and flexible sensor 1-4. Therefore flexible substrate with various electronic functions is highly demanded. Several researchers have conducted the fabrication of electronic devices on different substrates, such as silver resonator on ceramic ${ }^{5}$, copper circuit on nylon PA2200 6 , and copper nanostructure on sillicon $^{7}$. In order to fabricate metal film with flexible pattern design and great performance, various methods of metallization are developed. M. Farhan Shafique et al. used silver paste to coat the substrate first and then etch the unwanted part with laser ablation afterwards ${ }^{5}$. Matteo Calaon et al. fabricated a Al foil mask and deposited copper on the uncovered areas with physical vapour deposition (PVD) $\operatorname{method}^{8}$. Shih-Chun Huang et al. deposited copper film on polymer substrates with the aid of EDA ink by electroless deposition $^{9}$. Although these methods could finally realize the film metallization, it has involved quite complicated processing with the shortage of sufficient flexibility.

Electroless deposition is usually known as self-catalytic reduction of dissolved metal ions by reducing agent at the solid-liquid interface. Since 1950s, the electroless deposition techniques were rapidly developed for the growing market of printed circuits. Furthermore, it has been widely applicable for integrated circuits, decorative articles and semiconductors ${ }^{10}$. Commonly, electroless copper deposition on insulators, including plastics, glass and ceramics ${ }^{11}$, ${ }^{12}$, is considered as a chemically complex process mainly consisted of four steps: etching, neutralization, catalysis and electroless plating ${ }^{13}$.

Accordingly, it is desirable to explore a flexible and straightforward way to realize the fabrication of various patterns on non-conductive substrate. Laser activation as an alternative step, instead of etching, neutralization and catalysis, mainly reduces the processing complexity ${ }^{14}$. Meanwhile, laser processing as an environmental friendly and easy operation tool can achieve flexibly designed patterns $^{15,16}$. A lot of effort has been carried out mainly in three aspects. Firstly, the selection of electroless deposition solution could be extended. Milan Paunovic and Russ Arndt concluded that guanine and adenine can accelerate electroless copper deposition ${ }^{17}$. Fumihiro Inoue et al. studied copper bath stability by UV-Visible spectrum and $\mathrm{PH}$ measurement ${ }^{18}$. Secondly, the system setup could be intelligently optimized for laser processing equipment. Lothar Bauer developed an offline programming module for laser systems ${ }^{19}$. Fanhui Meng and Hongzhi Wang also developed a CAD/CAM software for laser micromachining ${ }^{20}$. Finally, substrates with blended catalytic seeds could be intendedly modified. The patent invented by LPKF Company demonstrated a modified plastic substrate by mixing organometallic complex ${ }^{21}$. However, few studies have been performed to optimize the laser processing parameters and understand the mechanism, which could have significant impact on the quality of deposited metal layer.

In this paper, we present a highly accurate and flexible method to fabricate confined copper layer on PC plastic by pulsed laser ablation. It involves the commercially improved grade of electroless deposition solution and modified PC plastic substrate. Etching, neutralization and catalysis (including stannum sensitization and palladium activation) process in conventional techniques have been automatically avoided. During the electroless deposition process, pulsed laser ablation is used to activate the surface of the PC plastic with specifically designed patterns. The modified PC plastic substrate contains active copper seeds, 
which serve as the self-catalytic centers. The surface morphologies of laser machined plastic and the copper layer after metallization are observed by scanning electron microscopy (SEM) to understand the process of copper deposition. This work could benefit systematic understanding of the tunable laser parameters effects on the film quality and electrical performance, thus further guide us to deposit copper layer under other non-conductive substrates by laser.

\section{Experimental}

Materials

The non-conductive PC plastic (HALCYON 5H003, ShenZhen) is used as substrate materials. With the premise of necessary mechanical and thermal properties, such as tenacity, hardness and melting point, $\mathrm{Cu}$ powder is mixed with the polycarbonate as much as possible. The PC substrate is formed by injection of the modified PC particles with a thickness $3 \mathrm{~mm}$. Electroless copper deposition is supported by the dissolved solution (STELLA SSC_ECU_18, Singapore). The chemicals named SCC_ECU_18_A mainly contain copper sulfate as copper ion provider and formaldehyde as the reducing agent. Solution SCC_ECU_18_B mainly contains sodium hydroxide which is used to control the $\mathrm{PH}$ of chemical solution.

\section{Selective activation by pulsed laser ablation}

Fig. 1 shows the process of laser selective activation of electroless copper deposition. The sample is prepared with the fixed size of $25 \mathrm{~mm} \times 40 \mathrm{~mm} \times 3 \mathrm{~mm}$. First of all, ultrasonicator is used to clean the PC plastic substrate with alcohol. The impurities generated from oil, ash or fingerprint could have complex and unexpected interaction with laser. Then it is of great importance to guarantee the cleanness of the plastic surface. Subsequently, the PC plastic surface is irradiated by pulsed fiber laser with 16 groups of combined parameters. The wavelength of the laser is $1064 \mathrm{~nm}$. The pulse duration is $100 \mathrm{~ns}$ and the repetition is $20 \mathrm{kHz}$. The spot size is $\sim 50 \mu \mathrm{m}$. The laser power is changed from 0.9 $\mathrm{W}$ to $1.4 \mathrm{~W}$ and scanning speed is changed from $450 \mathrm{~mm} / \mathrm{s}$ to $600 \mathrm{~mm} / \mathrm{s}$ in order to investigate their influence on copper deposition. An exhauster is used to pump air with vaporized plume. After laser irradiation, the substrate was treated again with alcohol to remove the residue on the PC plastic surface.

(a)

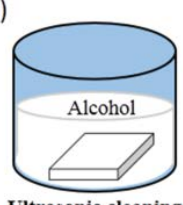

Ultrasonic cleaning

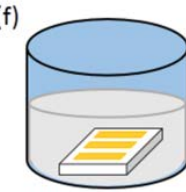

Antioxidant process

Fig.1 Schematic process of laser selective activation of electroless copper deposition.

\section{Copper layer deposition}

Fig. 1 also shows the schematic process of copper metallization. SCC_ECU_18_A and SCC_ECU_18_B are respectively mixed with the volume ratio of $3: 2$ and then mechanically agitated for 5 minutes. The mixed solution is evenly heated in a water bath. The temperature is raised to $\sim 50{ }^{\circ} \mathrm{C}$ by a fixed heating stage in order to speed up chemical reactions. It was observed that scattered brownish spots are on the surface at the very beginning and then the color of the surface gradually becomes darker. During this process, microbubbles could be observed. The thickness of the copper layer is related to the metallization time and usually the film can become electrically conductive in $\sim 2$ hours. Afterwards, the substrate is immersed with an antioxidant to inhibit the oxidation.

\section{Electrical measurement}

The resistance of each copper film is measured using 2wire Ohms measurement. The contact resistance is compensated. Each set of data is measured three times and then averaged to minimize the experimental errors.

\section{Material characterization}

Surface morphology is investigated by scanning electron microscope (Hitachi Su-70, Japan). Resistivity is measured by LCR meter (HIOKI IM3532, Japan).

\section{Results and discussion}

Morphology and chemical compositions

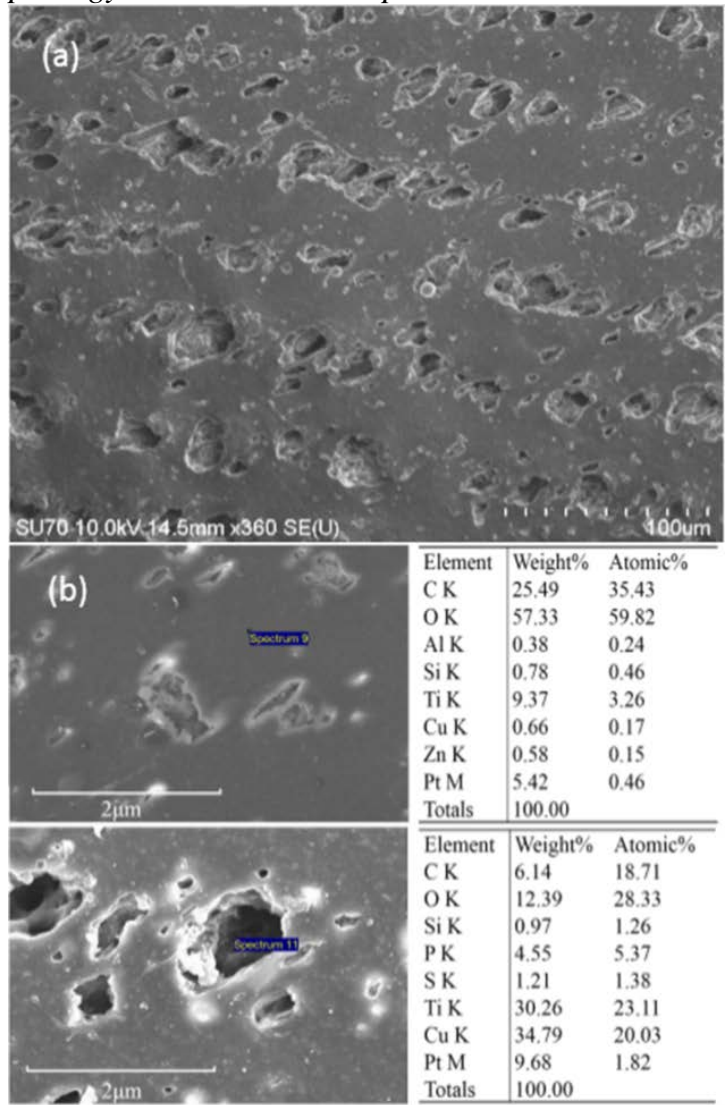

Fig.2 SEM images and EDX results of laser processed PC plastic substrate. 
The surface of PC plastic is fully composed of irregularly shaped holes and residues as shown in Fig.2(a). While PC plastic is irradiated by pulsed laser, the generated heat dissipates into the substrate by absorption of the pulsed laser and then the bulk temperature is raised up. The heataffected zone can change the surface morphology by recasting and formation of burrs. As the laser intensity reaches a certain level, the number of species within the plume exponentially increases by interaction with the laser beam which ionizes the molecules, leading to the formation of plasma plume. In the laser ablation, compressive forces can be created by the vapor and plasma plume at the laser focused point, leading to the expulsion of the molten pool of materials. The sputtered nanoparticles are solidified in air and then pushed away by the shock waves accompanied by plasma plume, which are finally absorbed by the exhauster. Attributed to the Gaussian distribution of the laser beam at the focus point, the vicinal area of laser ablation can absorb less energy with lower temperature, contrib- uting to the generation of smaller holes around central holes. Meanwhile, the chemical compositions of the PC plastic surface have been changed. Fig.2(b) shows the atomic percentages of various elements on the PC plastic surface before and after laser irradiation. Before pulsed laser ablation, the elements of $\mathrm{C}$ and $\mathrm{O}$ play the major roles and only $0.17 \% \mathrm{Cu}$ exists. Since copper powder is blended inside the PC plastic, $20.03 \%$ atomic percentage of $\mathrm{Cu}$ was detected in the laser irradiated area. Other elements, such as $\mathrm{Al}, \mathrm{Si}, \mathrm{Ti}, \mathrm{Zn}, \mathrm{P}$, are mixed up to enhance the thermodynamic properties of the PC plastic. The existence of $\mathrm{Cu}$ seeds provides the feasibility of electroless copper deposition.

\section{Evolution of copper deposition}

To investigate the mechanism of copper layer deposition on laser irradiated PC plastic surface, SEM is employed to observe the dynamic growth behaviors of deposited copper layer in different phases.
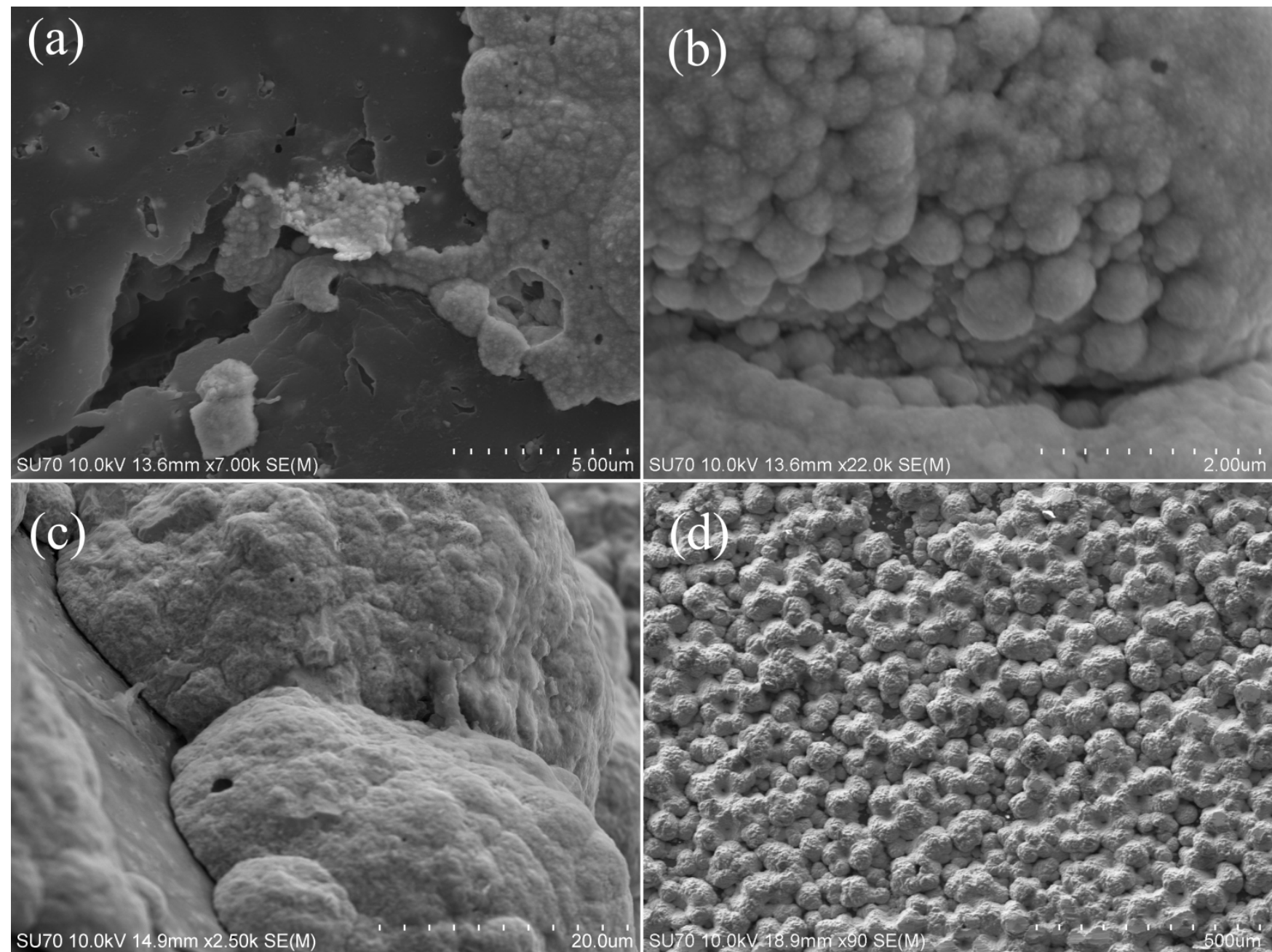

Fig.3 SEM images of copper deposition process in different periods: (a) copper emerging from laser created cracks on PC plastic surface;

(b) copper particles aggregate to copper islands; (c) bonding of copper islands; and (d) formation of copper film.

Figs.3(a-d) describe the dynamic process of the copper deposition. As shown in Fig.3(a), the width of the hole is $3 \mu \mathrm{m}$ and the length is $\sim 10 \mu \mathrm{m}$., The surface absorbs the short-duration laser pulse with concomitant electronic transitions from the ground singlet state to the first excited singlet state and broke the bond within the long-chain polymers ${ }^{22}$. The generated flow consists of gases $\left(\mathrm{CO}_{2}\right.$, $\mathrm{CO})$ and carbonaceous particles are ejected into air ${ }^{22,23}$. The dynamic process removes the surface materials and creates holes. The shape and size of the holes are determined by material physical and chemical properties, such as thermal conductivity coefficient, specific heat capacity, 
and mass density. It could be seen in Fig.3 that the copper layer grows from the inner of the laser ablated holes. The reliability of solid adhesion is strongly dependent on the embedded components. This process illustrates that pulsed laser can remove the superficial PC plastic materials and make $\mathrm{Cu}$ seeds exposed. These $\mathrm{Cu}$ seeds serve as catalytic centers of chemical reactions in the deposition process, aiding the formaldehyde in transporting electrons into cupric ions for reduction to form dehydrogenate and generate hydrogen. The holes inside the copper surface layer may be probably created by the generated hydrogen, which can significantly affect their mechanical properties.

While the copper layer grows out of the holes, the size of nanoparticles becomes larger. As shown in Fig.3(b), the copper nanoparticles aggregate by packing the smaller nanoparticles inside. The size of copper particles is confined in nano-scale level as the growth of copper layer. Based on the proposed mechanism, it could explain the morphologies and roughness of confined copper islands.

Fig.3(c) shows a bonding mechanism between isolated copper islands. Since there exists a short distance among holes created by pulsed laser, the copper islands initially grow up separately and then are gradually bonded to the neighbouring ones with formed copper bridges. Meanwhile, the growth of copper layer continues upon the copper islands, leading to more globular particles as shown in Fig. 3(d). These $\sim 50 \mu \mathrm{m}$ globular copper particles cover the copper islands and stack up layer by layer. The gaps and cavities between copper particles contribute to the porous structures of metal film, leading to the large specific surface area.

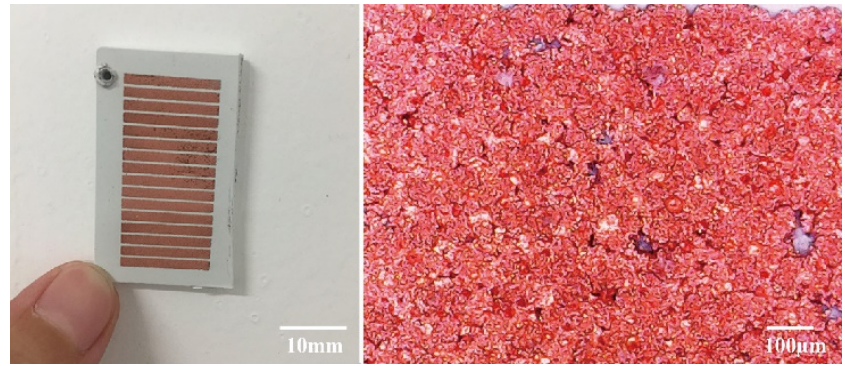

Fig.4 Copper films on PC substrate and its microscope images by tunable laser processing parameters.

Fig.4 shows the copper films on PC substrate and corresponding optical images. The width of each copper line is $1.2 \mathrm{~mm}$ and the length is $15 \mathrm{~mm}$. The laser activated areas are well deposited by copper layer without visible defects. It is easily observed that there is no copper deposited on non-activated areas. The quality of the deposited copper layer seems to be satisfactory, as the uniform density of film is confirmed by the optical image.

\section{Electrical properties of copper films}

Fig. 5 shows the cross-section of copper film on PC substrate. The bulk is PC plastic and the islands were copper particles. The thickness of the copper film is about 100 $\mu \mathrm{m}$.

The resistivity can be calculated based on the equation: $\rho=\mathrm{R} \cdot \mathrm{S} / \mathrm{L}$, where $\rho, \mathrm{R}, \mathrm{S}$ and $\mathrm{L}$ are the resistivity, resistance, cross section area and length of copper line, respectively.

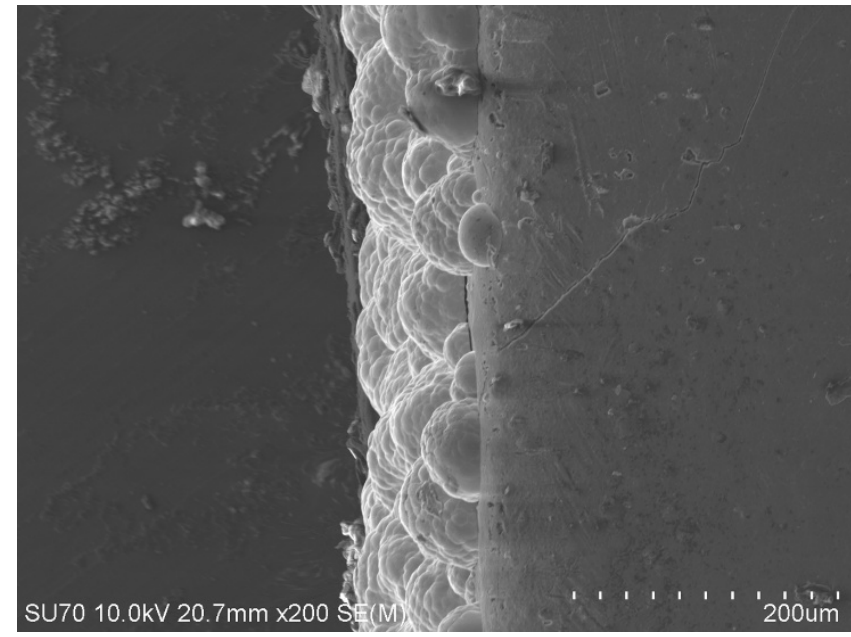

Fig.5 SEM cross section image of copper film.

Figs. 6 and 7 show the relationships among copper resistivity, laser power and scanning speed. As shown in the figures, the resistivity of fabricated copper films ranges from $15 \sim 44 \mu \Omega \cdot \mathrm{cm}$. The lowest resistivity of $15 \mu \Omega \cdot \mathrm{cm}$ is still 9 times higher than copper bulk $(1.72 \mu \Omega \cdot \mathrm{cm}$, $20{ }^{\circ} \mathrm{C}$ ). However, it shows significant improved conductivity compared to other groups' research on fabricating copper tracks on non-conductive substrates with different depostion or coating methods. For example, Siyuan Qi et al. fabricated copper paste with the resistivity of 75 $\mu \Omega \cdot \mathrm{cm}^{24}$. Sze Kee Tam synthesized copper paste with the of resistivity $58 \mu \Omega \cdot \mathrm{cm}^{25}$ and Cyuan-Jhang Wu et al synthesized copper paint with the resistivity of $774 \mu \Omega \cdot \mathrm{cm}$.

As shown in Fig.6, the peak of resistivity appears at the point of laser power $=0.9 \mathrm{~W}$, scanning speed $=500 \mathrm{~mm} / \mathrm{s}$ and the lowest point appears at laser power $=1.2 \mathrm{~W}$ and scanning speed $450 \mathrm{~mm} / \mathrm{s}$. It can be observed that the resistivity decreases with laser power based on the overall trend of curves. When pulsed laser is irradiated on the surface, the temperature is raised up at the interfacial zone between focusing point of laser beam and PC plastic surface, thermally decomposing the surface materials. As increasing laser power, it provides more thermal energy with each laser pulse, leading to faster temperature increase and larger heat affect zone on the focus point ${ }^{26}$. Larger heat affect zone could create larger holes and make more $\mathrm{Cu}$ seeds exposed. Since the $\mathrm{Cu}$ particles are distributed evenly on the PC plastic, laser beam with higher power could drill into deeper layers, leading to the decomposition of the polymer matrix and exposing $\mathrm{Cu}$ seeds from subsurface layer to deliver more opportunities for metallization.

It could be observed that there exists non-monotonous trend in the figures. The resistivity first increased with the speed increased from 450 to $500 \mathrm{~mm} / \mathrm{s}$ and then decreased with the speed increased from 500 to $550 \mathrm{~mm} / \mathrm{s}$ and again increased with speed increased from 550 to $600 \mathrm{~mm} / \mathrm{s}$. The lowest resistivity appears at scanning speed $550 \mathrm{~mm} / \mathrm{s}$ as shown in Fig.7. Estimated by scanning speed and repetition rate, the distance between each laser pulse is approximately $22.4 \mu \mathrm{m}$ to $30 \mu \mathrm{m}$. The density of the laser irradiated holes decreased with the speed, indicating that less exposure of $\mathrm{Cu}$ seeds count against copper growth. Therefore, the resistivity increased at first. 


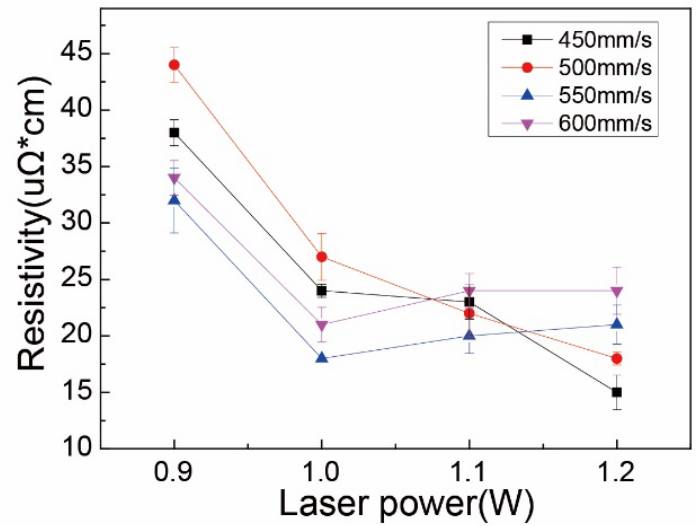

Fig.6 Relationship of the resistivity of copper films with laser power at different scanning speeds.

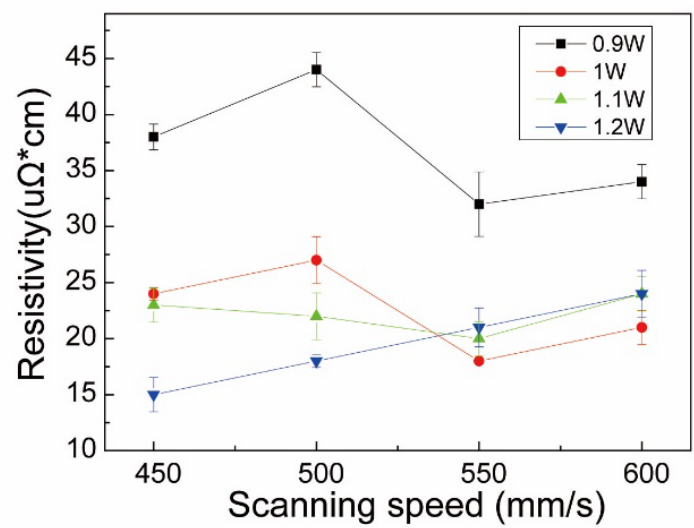

Fig.7 Relationship of the resistivity of copper films with scanning speed at different laser powers.

As the size of the laser spot is $\sim 50 \mu \mathrm{m}$, the laser pulses could be overlapped on the PC surface. After laser irradiation on the surface, carbonaceous burrs appear and adhere onto the roughened surface ${ }^{23,}{ }^{27}$. The surface chemistry, composition and nanostructures can be varied by the deposited carbonaceous dust, $\mathrm{Cu}$ seeds and deformation caused by laser, respectively. This means the overlapping of the laser pulses would affect the surface activity for electroless deposition. The areas of overlapped region decreased with the scanning speed. It may explain why the resistivity decreased on speed 500-550 mm/s. Nevertheless, in the range of higher scanning speed, the resistivity increased again, which may be attributed to the density of pulses. The processing effect could be optimized for laser activated PC plastic substrate with scanning speed around 450 550 $\mathrm{mm} / \mathrm{s}$ and laser power around $1 \mathrm{~W}$ in a narrow range.

\section{Mechanism of copper layer deposition}

The copper layer deposition is realized by the electroless deposition technique. Copper electroless solutions were firstly reported by Narcus ${ }^{28}$ in 1947. A further complete and commercial technique was reported by Cahill using formaldehyde as reducing agent ${ }^{29}$. It works as a selfcatalytic process. The copper nanoparticles initially deposited on the selectively activated surface and continuously assemble layer by layer The catalysis process in electroless deposition was studied by Haruyama and $\mathrm{Ohno}^{30}$ and by Wiese and $\mathrm{Weil}^{31}$. Van Den Meerakkeer also proposed a mechanism, which demonstrated that the dehydrogenation step of formaldehyde determines the catalytic nature of electroless copper solutions ${ }^{32}$.

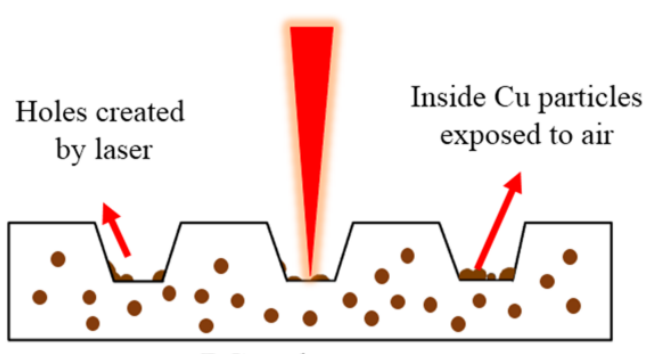

PC substrate

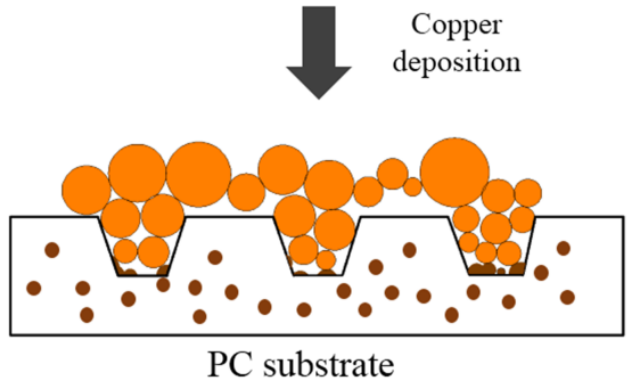

Fig. 8 Schematic mechanism of copper film deposition on PC plastic surface.

The electroless plating of copper layer is understood as cathodic reduction of cupric ions and anodic oxidation of formaldehyde simultaneously at the copper surface based on the widely accepted mixed potential theor $y^{33}$. On the copper surface, cupric ions capture electrons supplied by formaldehyde and deposited as $\mathrm{Cu}^{0}$.

Cathodic reaction,

$\mathrm{Cu}^{2+}+2 e^{-} \rightarrow \mathrm{Cu}^{0}$

Anodic reaction,

$2 \mathrm{HCHO}+4 \mathrm{OH}^{-} \rightarrow 2 \mathrm{HCOO}^{-}+\mathrm{H}_{2} \uparrow+2 \mathrm{H}_{2} \mathrm{O}+2 e^{-}$

Over all reaction,

$\mathrm{Cu}^{2+}+2 \mathrm{HCHO}+4 \mathrm{OH}^{-} \stackrel{\mathrm{CAT}}{\rightarrow} \mathrm{Cu}^{0}+2 \mathrm{HCOO}^{-}+2 \mathrm{H}_{2} \mathrm{O}+\mathrm{H}_{2}$

In this work, the PC plastic as an insulator should be pre-processed in advance. Laser ablation as an effective activation approach is applied to selectively remove the polymeric materials and make $\mathrm{Cu}$ seeds exposed. Fig.8 is the schematic drawing of copper deposition process. The exposed $\mathrm{Cu}$ seeds in the laser generated holes serve as catalytic centers, facilitating HCHO to release electrons. The absorbed $\mathrm{Cu}^{2+}$ ions are reduced as $\mathrm{Cu}^{0}$ and then deposited on the $\mathrm{Cu}$ seeds exposed from PC plastic. Subsequently, the deposited $\mathrm{Cu}$ nanoparticles become the new catalytic centers surrounded by deposited $\mathrm{Cu}$ nanoparticles. The copper nanoparticles grow out of the holes and are bonded to neighboring copper particles.

During the laser activation process, single pulse energy is determined by laser power and repetition rate. Lower energy may not insufficiently tailor the polymer materials on PC surface and fail to make $\mathrm{Cu}$ seeds exposed. On the other hand, higher energy would probably cause polymeric 
materials to be burnt out. Meanwhile, the distance between holes determined by the scanning speed and repetition rate plays an important role. In the case of larger distance, the copper islands may not be able to bond to each other and the further deposition is inhibited. It is also noted that a slower scanning speed would cause laser activated region overlapped and accumulate enormous heat to make the surface damaged.

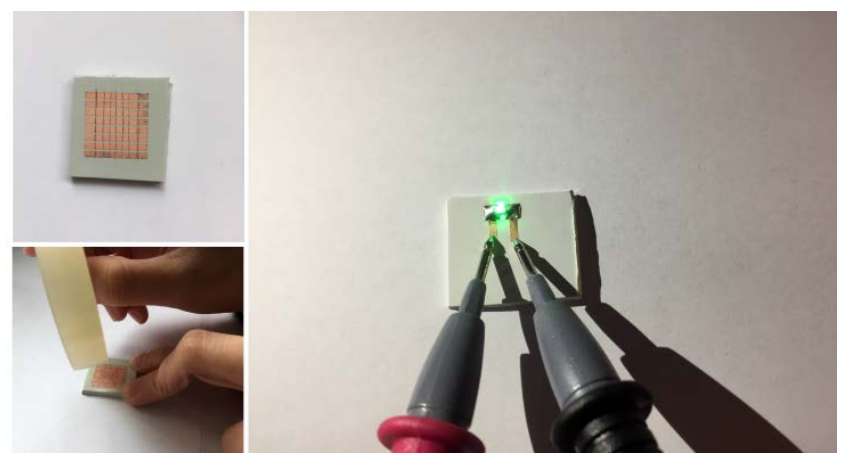

Fig.9 Adhesion testing and LED light connected to the copper conductive tracks.

The testing of deposition layer adhesion was carried out according to GB5270-85. Firstly, a knife is used to cut the film into pieces with the size of $2 \mathrm{~mm} \times 2 \mathrm{~mm}$ and then the tap is pasted followed by the process of quick tearing-off. It shows that the deposited copper film could not be removed by $3 \mathrm{M}$ tap (Scotch Magic tape 3M600). The LED emission image indicates good conductivity and adhesion of the copper films. The size of the copper lines is $1.5 \mathrm{~mm} \times 8$ $\mathrm{mm}$ and the packing of LED is 0805 .

\section{Conclusions}

In summary, a flexible and environmental friendly method is developed to fabricate confined copper film on non-conductive modified PC plastic. Laser activation process can assist the exposure of the catalytic seeds for electroless deposition. The thickness of the film is about 100 $\mu \mathrm{m}$ and the resistivity of $15 \mu \Omega \cdot \mathrm{cm}$ could be achieved by tuning the laser activation parameters. The influence of the laser power and scanning speed on film resistivity are investigated. The growth mechanism of the copper film is systematically studied. A process model of the copper deposition is proposed to describe the dynamic process of electroless copper deposition, which could explain the morphology of copper film and its effect on electrical properties.

\section{Acknowledgements}

This research is supported by the National Natural Science Foundation of China (No.61605162), the National Basic Research Program of China (No.2013CBA01703), Natural Science Foundation of Fujian Province of China (No. 2017J05106) and Collaborative Innovation Center of High-End Equipment Manufacturing in Fujian. The authors also thank Stella Specialty Chemicals Pte Ltd for the supply of SSC_ECU_18.

\section{References}

1. A. Islam, H. N. Hansen and N. Giannekas: CIRP Ann. Manuf. Technol., 64, (2015) 539.
2. D. H. Kim, N. Lu, R. Ma, Y. S. Kim, R. H. Kim, S. Wang, J. Wu, S. M. Won, H. Tao, A. Islam, K. J. Yu, T. i. Kim, R. Chowdhury, M. Ying, L. Xu, M. Li, H. J. Chung, H. Keum, M. McCormick, P. Liu, Y. W. Zhang, F. G. Omenetto, Y. Huang, T. Coleman and J. A. Rogers: Science, 333, (2011) 838.

3. T. Sekitani, T. Yokota, U. Zschieschang, H. Klauk, S. Bauer, K. Takeuchi, M. Takamiya, T. Sakurai and T. Someya: Science, 326, (2009) 1516.

4. T. Leneke, H. Soeren and B. Schmidt: ESTC, (2008) 663.

5. M. F. Shafique, K. Saeed, D. P. Steenson and I. D. Robertson: IEEE Trans. Microwave Theory Tech., 57, (2009) 3254.

6. P. Amend, C. Pscherer, T. Rechtenwald, T. Frick and M. Schmidt: Physics Procedia, 5, (2010) 561.

7. S. M. Huang, M. H. Hong, Y. F. Lu, B. S. Lukỳanchuk, W. D. Song and T. C. Chong: J. Appl. Phys., 91, (2002) 3268.

8. M. Calaon, A. Islam, H. N. Hansen and C. Ravn: Int. J. Adv. Manuf. Technol., 59, (2012) 101.

9. S. C. Huang, T. C. Tsao and L. J. Chen: J. Electrochem. Soc., 157, (2010) D222.

10. P. Balaramesh: Int. J. Innov. Res. Stud., 3(4), (2014) 167.

11. J. Kim, S. H. Wen, D. Y. Jung and R. W. Johnson: IBM J. Res. Dev., 28, (1984) 697.

12. X. Cui, D. A. Hutt and P. P. Conway: Thin Solid Films, 520, (2012) 6095.

13. G. A. Krulik: J. Chem. Educ., 55(6), (1978) 361.

14.Y. Zhang, H. N. Hansen, A. De Grave, P. T. Tang and J. S. Nielsen: Int. J. Adv. Manuf. Technol., 55, (2010) 573

15. T. C. Chong, M. H. Hong and L. P. Shi: Laser Photonics Rev., 4, (2010) 123.

16. M. H. Hong, S. M. Huang, B. S. Luk'yanchuk and T. C. Chong: Sens. Actuators, A, 108, (2003) 69.

17. M. Paunovic: J. Electrochem. Soc., 130, (1983) e309.

18.F. Inoue, H. Philipsen, A. Radisic, S. Armini, Y. Civale, S. Shingubara and P. Leunissen: J. Electrochem. Soc., 159, (2012) 4.

19. L. Bauer: Offline Programming of 3D Laser Systems, 2787, (1996) 34.

20. M. Fanhui and W. Hongzhi: 11th International Conference on Electronic Packaging Technology \& High Density Packaging (ICEPT-HDP), (2010) 902.

21. U. S. Pat., U. S. 6,319,564.20, (2001).

22. M. A. Roberts, J. S. Rossier, A. Paul Bercier and H. Girault: Anal. Chem., 69, (1997) 2035.

23. F. Caiazzo, F. Curcio, G. Daurelio and F. M. C. Minutolo: J. Mater. Process. Technol., 159, (2005) 279.

24. S. Qi, R. Litchfield, D. A. Hutt, B. Vaidhyanathan, C. Liu, P. Webb and S. Ebbens: IEEE 62nd Electronic Components and Technology Conference, (2012) 1651.

25. S. K. Tam and K. M. Ng: J. Nanopart. Res., 17, (2015) 1.

26. A. K. Ghavidel, M. Shabgard and H. Biglari: J. Appl. Polym. Sci., 133, (2016) 1.

27. P. A. Atanasov: Proc. SPIE Int. Soc. Opt. Eng., 3092, (1997) 772.

28. H. Narcus: Met. Finish., 45, (1947) 964.

29. A. E. Cahill: Am. Electrochem. Soc. Proc., 44, (1957) 130. 
30. I. O. S. Haruyama: Electroless Deposition of Metals and Alloys, 12, (1988) 20.

31. H. Wiese: Electroless Deposition of Metals and Alloys, 12, (1988) 53.

32.V. D. Meerakker: J. Appl. Electrochem., 11, (1981) 395.

33. M. Paunovic: Plating, 55, (1968) 1161.

(Received: April 25, 2017, Accepted: September 3, 2017) 